\title{
EFFECT OF PAEDIATRIC FLAT FOOT ON THE SAGITTAL ALIGNMENT OF THE PELVIS AND SPINE
}

\section{Elena Adelina PANAET ${ }^{*}$, Vasilica GRIGORE ${ }^{1}$, Maria Cristina MAN ${ }^{2}$, Cristina Mihaela DOLINSCHI ${ }^{3}$, Dan Iulian ALEXE ${ }^{4}$}

\footnotetext{
${ }^{1}$ National University of Physical Education and Sport, Faculty of Physical Education and Sport, Bucharest, Romania

2 "1 Decembrie 1918" University, Alba Iulia, Romania

${ }^{3}$ National Institute of Aeronautical and Space Medicine, Bucharest, Romania

4 "Vasile Alecsandri" University, Bacău, Romania

*Corresponding author: adelina_panaet@yahoo.com
}

https://doi.org/10.35189/dpeskj.2021.60.4.10

\begin{abstract}
Human body segments interact harmoniously and efficiently so that homeostasis is maintained in any circumstances. Even the slightest modification of the support surface can have repercussions on postural control strategies and consequently can lead to a change in the alignment of the whole body. Flat foot is the most common condition of the lower limb. It is clinically manifested on multiple anatomical planes: flattening of the medial longitudinal arch (sagittal plane), valgus of the calcaneus (frontal plane) and abduction of the anterior foot (transverse plane). In any case, the influence of structural modifications of the flat foot on one's posture, especially in children, still remains an insufficiently researched topic. The purpose of this study was to assess the effects that structural modifications of a flat foot have on the pelvis and spine in the sagittal plane, in children aged 7-9. Thus, the following hypothesis was formulated: the structural modifications of flat feet can modify the sagittal alignment of the pelvis and spine in children aged 7-9. The flat feet were assessed using the Foot Posture Index-6 (FPT-6) while the sagittal alignment of the pelvis and spine was measured using the GPS400 computerised postural analysis system. Statistical analysis of the data did not indicate any significant correlations between the FPI-6 score and pelvic tilt angle or between the FPI-6 score and differences between the sensors and the lumbar and dorsal spinous processes.
\end{abstract}

Keywords: flat foot, pronation, pelvis, spine, posture.

\section{Introduction}

The plantar surface, which is rich in somatic-sensory afferentations, provides information that allows the adjustment of postural responses (Alexe, 2013). Thus, even the slightest modification of the support surface can have repercussions on postural control strategies and consequently can lead to a change in the alignment of the whole body. Cordun (2009) also believes that the asymmetrical distribution of plantar pressure causes a change in the architecture of the foot. Therefore, this could be the starting point for upper-muscle chain imbalances that alter postural alignment.

Flat foot is the most common condition of the lower limb. Several authors tried to define flat foot by including two consistent features: the collapse of the medial longitudinal arch and the valgus heel position. It can occur in one foot (unilateral) or in both feet (bilateral). Most epidemiological studies have shown that flat foot has a high prevalence. For the 7-12 age group, Chang et al. (2010) identified 1,222 (59\%) flatfoot cases in a sample of 2,083 children, mostly among 8 -year-olds. It was noticed that boys were more affected by this condition compared to girls. Specialists in the field record the excessive eversion of the subtalar joint as 
a consistent characteristic of the flat foot. According to this, the following changes may occur in the structure of the foot: plantar flexion of the talus, plantar flexion of the calcaneus in relation to the tibia (equine posture), dorsal flexion and abduction of the navicular, valgus posture of the heel, abduction and supination of the forefoot (Ezema et al., 2013). Moreover, a collapse of the posterior face of the navicular can be observed radiologically, which creates an expansion of the articular space with the talus (Peeters et al., 2013). These changes trigger the collapse of the medial longitudinal arch (Dare \& Dodwell, 2014).

In reality, the structural changes of the flat foot are manifested more than by a simple diminution of the medial longitudinal arch or the valgus posture of the heel. In some clinical cases, the medial edge of the foot becomes convex and causes full contact with the support surface. As a result, excessive tension is triggered in the deltoid ligament and posterior tibial muscle, whose functions are inhibited. These changes and the valgus posture of the hindfoot disrupt the functioning of the midfoot and forefoot. From this point, the upper segments are forced to develop compensatory responses in order to both solve the problems imposed by the adoption of this posture and maintain balance.

It has been postulated that the flattened appearance of the foot is potentially problematic. Several studies have tested the link between the type of foot (supine or prone) and the alignment of the lower limbs and pelvis. In a closed cinematic chain, studies suggest that there are a series of modifications caused by the pronation of the subtalar joint (a component of the flat foot). Characterised by the adduction, plantar flexion and eversion of the calcaneus, the pronation of the subtalar joint can cause a medial and inferior translation of the talus. Its position in relation to the tibia and fibula forces the tibia to perform an internal rotation motion (Tiberio, 2000). The knee joins the tibia and spins in the same direction (only under static conditions). As a result, an internal rotation of the hip is generated, which pushes the femoral head in the posterior acetabulum, causing the pelvis to move forward (Khamis \& Yizhar, 2007). Thought to be a key element between the upper and lower body, the position of the pelvis can influence the spine through the anatomical connections between the two segments. Moreover, this adjustment, which can affect the lumbar spine, causes a deviation of the centre of mass from the ideal postural alignment. As a result, the body will use strategies of postural compensation to bring back the centre of mass projection within the base of support (Ghasemi et al., 2016).

In any case, the influence of structural modifications of the flat foot on the pelvis and spine posture in the sagittal plane, especially in children, still remains an insufficiently researched topic.

The purpose of this study was to assess the effects that structural modifications of a flat foot have on the pelvis and spine in the sagittal plane, in children aged 7-9.

Thus, the following hypothesis was formulated: the structural modifications of flat feet can modify the sagittal alignment of the pelvis and spine in children aged 7-9. 


\section{Methodology}

\section{Participants}

The participants were selected using the Foot Posture Index-6 (FPI-6). Thus, out of a total number of 64 participants proposed by an interdisciplinary team, 51 children aged between 7 and 9 (7.54 \pm 0.61 years) were assessed with FPI-6.

Based on the results recorded using the FPI-6, 23 children $(7.43 \pm 0.58$ years old $)$ were proposed for postural analysis. After being presented the purpose of the research, the children's parents/legal guardians accepted their participation in the study (Figure 1).

The inclusion criteria were: age between 7 and 9, an FPI-6 score higher than 6 points, flexible flat foot. The exclusion criteria were: rigid flat foot, coexisting musculoskeletal pathologies of the foot and ankle, neurological pathologies, previous surgery of the lower limb and spine, use of plantar orthoses or supports.

\section{1 participants (assessed with FPI-6)}

23 participants identified with flat feet and proposed for postural analysis

Figure 1. General presentation of the process of selecting the participants included in the preliminary study

\section{Instruments}

a. Assessment of the type of foot

Foot Posture Index-6 (FPI-6) is a method used to assess foot posture based on preestablished morphological and functional criteria. This method uses a simple numeric scale to assess quantitatively and qualitatively the foot posture in the standing position. FPI-6 is a clinical instrument used to quantify how much a foot is in pronation, is neutral (normally developed) or in supination. When the assessor has time and materials, it is not recommended to use the Foot Posture Index to assess the patient's gait. Still, studies have shown that FPI-6 is a more valid approach in comparison with many of the static and non-weighing goniometric methods that are currently used in clinical evaluation (Evans et al., 2003; Menz, 1998; Redmond et al., 2006).

The raw value of the Foot Posture Index for each foot is calculated by adding the raw values of the morpho-functional indices for the 6 markers:

1) palpation of the talar head;

2) curvatures above and below the lateral malleolus;

3) inversion/eversion of the calcaneus;

4) prominence of the talonavicular joint; 
5) shape of the medial longitudinal arch;

6) the abduction/adduction of the anterior foot.

b. Global postural analysis

Postural analysis was conducted using the Global Postural System 400 - GPS (Chinesport FISIOTERAPIA). This device uses non-invasive techniques and methods to assess body alignment. It comprises multiple components and a software program that calculates different variables related to body morphology. GPS 400 software runs in English on any computer equipped with the Windows operating system. It scans the body, collects information and takes pictures in real time. It allows the recording of personal data and the comparison of analyses after a period of time to track the results obtained. The sensor highlights the following:

- horizontal axis of the eyes;

- nose-ears axis;

- head position;

- shoulder position;

- the line created by the inferior angle of the shoulder blades;

- pelvis line;

- knee posture;

- ankle posture;

- the degree of rotation of the body.

\section{Procedure}

a. Foot assessment using the FPI-6 - to determine the type of foot

Each participant was instructed to stand barefoot on a wooden structure with the following dimensions: $40 \mathrm{~cm}$ wide x $44 \mathrm{~cm}$ long x $20 \mathrm{~cm}$ high. Children were instructed to stand in a relaxed position while looking at a target $10 \mathrm{~m}$ away, mounted to a wall 2 metres above the floor. They were also asked not to move during the assessments.

This way, the anatomical markers set in the FPI-6 were assessed, recording a score between -2 and +2 , depending on the type of foot. The results were analysed as follows: 0 and $+5=$ neutral foot; between +6 and $+9=$ foot in pronation; $+10=$ foot in pronounced pronation; between -1 and $-4=$ foot in supination; $-5=$ foot in pronounced supination.

b. Posture assessment - GPS 400

The posture was analysed using the Lux Postural Analyser. The temperature of the room where the tests were conducted was previously raised to optimal levels. The surfaces were constantly washed and disinfected.

Children were asked to undress and stand on the plantar marks according to the indications on the surface of the platform. The system scanned the body, collected the information and took pictures in real time. The sensors on the gliding wires of the platform frame measured pelvic tilt angle and distances between lumbar and dorsal spinous processes in relation to the reference point of the device. 
A series of statistical indices were determined with regard to the Foot Posture Index score (for the right foot: FPI-6-rf and left foot: FPI-6-lf), pelvic tilt angle and distances between the lumbar and dorsal spinous processes and the posterior sensor.

Pearson's correlation coefficient was calculated to establish the relationship and its intensity between the variables. Independent variables - IVs (played the role of IVs) were represented by the right and left FPI scores (FPI-6-rf, FPI-6-lf), while dependent variables (DVs) were represented by: pelvic tilt angle, lumbar spinous process - posterior sensor distance, dorsal spinous process - posterior sensor distance.

\section{Results}

Table 1 and Figure 2 summarise the descriptive indices for the distribution of FPI-6 raw values for the right foot and left foot. The values were calculated for each participant.

Table 1. Descriptive indices for the distribution of FPI-6 values

\begin{tabular}{lcc}
\hline \multicolumn{1}{c}{ Statistical markers } & FPI-6 score - right foot & FPI-6 score - left foot \\
\hline Minimum & 6 & 7 \\
Maximum & 12 & 12 \\
$\mathrm{~m}$ & 9.78 & 9.78 \\
Median & 10 & 10 \\
$\mathrm{~s}$ & 1.62 & 1.41 \\
\hline Note: $\mathrm{m}=$ arithmetical mean; $\mathrm{s}=$ standard deviation; FPI-6 = Foot Posture Index-6
\end{tabular}

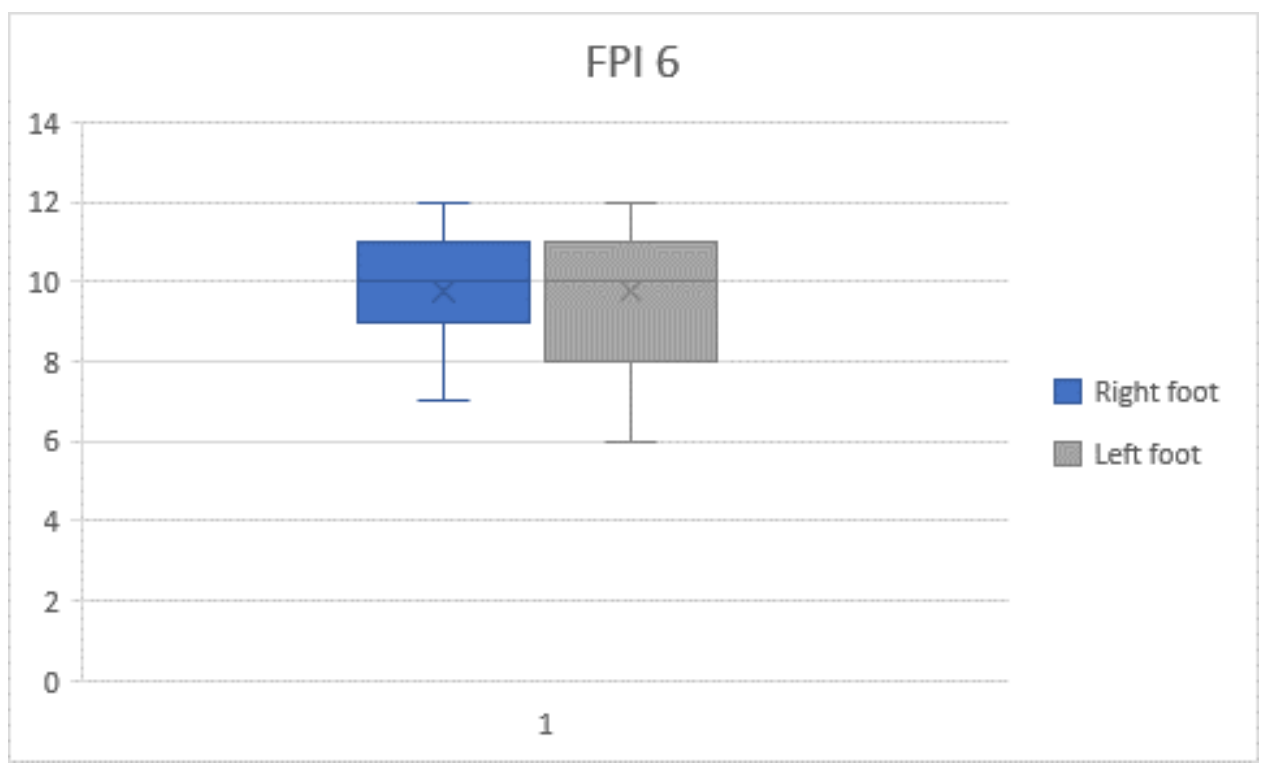

Figure 2. FPI-6-rf score; FPI-6-lf score

In the case of the distribution of children with flat foot based on the FPI- 6 score for the right foot and left foot (Figure 2), preliminary data analysis shows that there are no extreme values (Predoiu, 2021), considering both scores (FPI-6-rf and FPI-6-lf). Also, the result distribution for the right foot and left foot indicated an asymmetry with a crowding towards high values. As one can see in Table 1 and Figure 2, in both cases (FPI-6-rf and FPI-6-lf), the 
median of the series had the value 10 , meaning that half of the participants were below/above this value. There is a decreased concentration of the FPI-6-lf score compared to FPI-6-rf in the lower side of the figure. Interpretation of the FPI-6 score shows that the value of a score $\geq$ 10 represents a foot in pronounced pronation.

Table 2. Descriptive indices for the distribution of values of dependent variables

\begin{tabular}{lccc}
\hline Statistical markers & $\begin{array}{c}\text { Pelvic tilt angle } \\
\text { (degrees) }\end{array}$ & $\begin{array}{c}\text { Lumbar spinous process - posterior } \\
\text { sensor distance }(\mathrm{cm})\end{array}$ & $\begin{array}{c}\text { Dorsal spinous process - } \\
\text { posterior sensor distance }(\mathrm{cm})\end{array}$ \\
\hline Minimum & 4 & 17.32 & 16.52 \\
Maximum & 9.10 & 24.06 & 22.54 \\
$\mathrm{~m}$ & 7.09 & 21.76 & 19.30 \\
Median & 7.10 & 21.71 & 18.86 \\
$\mathrm{~s}$ & 1.24 & 1.73 & 1.53 \\
\hline Note: $\mathrm{m}=$ arithmetical mean; $\mathrm{s}=$ standard deviation; $\mathrm{cm}=$ centimetres
\end{tabular}

Pearson's correlation coefficient for FPI-6-rf and FPI-6-lf scores and dependent variables

The results indicate that there are no significant correlations between FPI-6-rf and FPI-6-lf scores and dependent variables (Table 3).

Table 3. Pearson's correlation coefficient for independent and dependent variables

\begin{tabular}{lcccc}
\hline & Pelvic tilt angle & $\begin{array}{c}\text { Dorsal spinous process - } \\
\text { posterior sensor distance }\end{array}$ & $\begin{array}{c}\text { Lumbar spinous process - } \\
\text { posterior sensor distance }\end{array}$ \\
\hline FPI-6 - rf & $\mathrm{r}$ & -0.035 & -0.318 & -0.154 \\
score & $\mathrm{p}$ & 0.874 & 0.139 & 0.482 \\
& $\mathrm{~N}$ & 23 & 23 & 23 \\
FPI-6 - lf & $\mathrm{r}$ & 0.069 & 0.081 & 0.099 \\
score & $\mathrm{p}$ & 0.753 & 0.714 & 0.652 \\
& $\mathrm{~N}$ & 23 & 23 & 23 \\
\hline
\end{tabular}

\section{Pearson's correlation coefficient for dependent variables}

A positive significant correlation with a strong intensity - $r^{2}$ was found (Predoiu, 2020) between two dependent variables: lumbar spinous process - posterior sensor distance and dorsal spinous process - posterior sensor distance $(\mathrm{r}=0.774, \mathrm{p}<0.000)$. There are no significant correlations between pelvic tilt angle and the other two dependent variables (Table 4).

Table 4. Pearson's correlation coefficient for dependent variables

\begin{tabular}{lcccc}
\hline & & Pelvic tilt angle & $\begin{array}{c}\text { Dorsal spinous process - } \\
\text { posterior sensor distance }\end{array}$ & $\begin{array}{c}\text { Lumbar spinous process - } \\
\text { posterior sensor distance }\end{array}$ \\
\hline Pelvic tilt angle & $\mathrm{r}$ & 1 & 0.067 & 0.327 \\
& $\mathrm{p}$ & & 0.763 & 0.128 \\
& $\mathrm{~N}$ & 23 & 23 & 23 \\
Dorsal spinous & $\mathrm{r}$ & & 1 & 0.774 \\
process - posterior & $\mathrm{p}$ & & & 0.000 \\
sensor distance & $\mathrm{N}$ & & 23 & 23 \\
\hline
\end{tabular}




\section{Discussion}

Considering the inter-segmental anatomical and biomechanical collaboration and the importance of the feet in developing and adjusting postural responses, the authors started from the assumption that any foot modification could cause compensatory movements in the upper body. In this sense, the following hypothesis was formulated: the structural modifications of flat feet can modify the sagittal alignment of the pelvis and spine in children aged 7-9. The results did not record any data to confirm this hypothesis.

Flat feet and sagittal pelvic tilt

The results of this study suggest that there are no statistically significant correlations between FPI-6 and sagittal pelvic alignment.

Under laboratory conditions, Khamis and Yizhar (2007) used inclined surfaces to induce bilateral eversion of the calcaneus. Their results showed that an increase of 10 degrees in the inclination angle of the support surface caused a calcaneal eversion of 1-3 degrees and a pelvic modification of 0.5 degrees on average. These authors have concluded that anterior sagittal pelvic tilt can be attributed to standing hyper-pronation. Relying on the results of the previous study, Pinto et al. (2008) showed that the bilateral pronation of the foot caused changes of 1.57 degrees (SD 1.57; 95\% CI $=0.70-2.49 ; \mathrm{F}=13.844 ; \mathrm{p}=0.003$ ) in the anterior pelvic tilt.

In contrast, Duval et al. (2010) did not record any results that could establish a statistical link between the pronation of the feet and the forward movement of the pelvis $\left(R=0.30, R^{2}\right.$ $=0.09$ ). Similarly, Khamis et al. (2015) did not record any data to confirm their hypothesis. The authors presumed that sagittal pelvic tilt could be caused by the pronation of the feet $(\mathrm{r}=$ $-0.052, \mathrm{p}=0.76$ ). Inducing hyper-pronation by inclining the support surfaces is likely to cause compensatory mechanisms able to prevent changes in the pelvic and lumbar spine alignment, as the test time is too short.

\section{Flat feet and sagittal alignment of the spine}

Based on the anatomical link between foot, leg, pelvis and spine, the professional literature suggests the following: the functionality of the feet may be perturbed in the case of flat feet; bilateral pronation of the subtalar joint (a component of the flat foot) can lead to biomechanical adjustments manifested by an internal rotation of the tibia, an internal rotation of the femur and a forward motion of the pelvis (Ghasemi et al., 2016); the pelvis, as a link between the lower and upper body, could cause the spine to modify its alignment in order to restore postural balance (Legaye et al., 1998).

The results of our study did not find any correlations between the FPI-6 score (flat foot highly pronated) and the alignment of the lumbar or dorsal spine. One could argue that the lower limbs have already performed compensatory movements to restore postural balance before reaching the pelvis.

Confirming our study results, data from Duval et al. (2010) suggest that the foot eversion (surrogated with the pronation of the subtalar joint) does not influence the position of the pelvis $\left(\mathrm{R}=0.30, \mathrm{R}^{2}=0.09\right)$ and spine $\left(\mathrm{R}=0.05, \mathrm{R}^{2}=0.003\right)$. The aforementioned researchers think that the manipulation of ankle movements was too small to have an effect on the pelvis and lumbar spine. More than that, their results do not indicate any statistical links between the degree of pelvic tilt and the modification of lumbar curvature $(R=0.04, p$ 
$=0.68)$. The authors of that study believe that the connective tissue between the spine and the pelvis allows a certain independence of movement and, as a result, no postural responses are being triggered.

However, the data from our study indicate a significant positive correlation of strong intensity between the lumbar spinous process - posterior sensor distance and the dorsal spinous process - posterior sensor distance $(r=0.774, \mathrm{p}<0.000)$. According to Giglio and Volpon (2007), the sagittal spinal curvature tends to increase linearly with age in a wellrepresented range, with no gender differences.

Farokhmanesh et al. (2014) stated in their study that dorsal kyphosis appeared as a compensatory response to the exacerbation of lordotic curvature. Compared to our study, the authors mentioned above found significant correlations between the bilateral pronation of the feet and the change in the lumbar and dorsal spine alignment. The inclination of support surfaces with 10, 15, 20 degrees modified the curvatures with 1.9, 2.2 and 2.4 degrees, and $0.9,1.4$, and 1.4 degrees, respectively. Similar results were also obtained in a study by Ghasemi et al. (2016). These authors concluded that hyper-pronation produced significant modifications in the sacrum, lumbar spine and dorsal spine.

In contrast to previous results, Clément et al. (2013) did not find any correlation between lumbar lordosis and thoracic kyphosis when they divided the sample of participants into two groups according to the value of thoracic kyphosis. These authors state that the alignment of thoracic spine is related to the alignment of lumbar spine in the sagittal plane, but this depends on the apex of lumbar lordosis, which can be altered by the pelvic parameters.

\section{Limitations and other considerations}

Firstly, in our research, we cannot establish a clear causal relationship between the flat foot and other variables. Longitudinal research is needed for a better understanding of this issue. Secondly, the method by which we can measure the modification of the spine alignment may not be sensitive enough to allow extracting accurate data. Another limitation is possibly due the small number of participants.

\section{Conclusion}

The purpose of this study was to assess the effects that structural modifications of a flat foot have on the pelvis and spine in the sagittal plane, in children aged 7-9. The results of our study suggest that there are no significant statistical correlations between FPI-6 and the sagittal alignment of the pelvis and spine. We have found no evidence that there is a relationship between the pronation or hyper-pronation of the feet (scored with FPI-6) and the anterior pelvic tilt. However, we cannot establish a relationship between the pronation or hyper-pronation of the feet and the lumbar or dorsal spine. We assume that, if the manipulation of the foot posture does not have a significant effect on the pelvis, then it cannot change the sagittal alignment of the spine either. This may be due to the lower limbs, which have already performed compensatory movements to restore postural balance before reaching the pelvis. 


\section{References}

Alexe, D. I. (2013). Echilibrul la pubertate. Relația dominanță emisferică-performanță [Balance during puberty. The hemispheric dominance-performance relationship]. Performantica.

Chang, J.-H., Wang, S.-H., Kuo, C.-L., Shen, H. C., Hong, Y.-W., \& Lin, L.-C. (2010). Prevalence of flexible flatfoot in Taiwanese school-aged children in relation to obesity, gender, and age. European Journal of Pediatrics, 169(4), 447-452. https://doi.org/10.1007/s00431-009-1050-9

Chinesport FISIOTERAPIA. https://www.chinesport.it/

Clément, J.-L., Geoffray, A., Yagoubi, F., Chau, E., Solla, F., Oborocianu, I., \& Rampal, V. (2013). Relationship between thoracic hypokyphosis, lumbar lordosis and sagittal pelvic parameters in adolescent idiopathic scoliosis. European Spine Journal, 22(11), 2414-2420. https://doi.org/10.1007/s00586-013-2852-z

Cordun, M. (2009). Kinantropologie [Kinanthropology]. CD Press.

Dare, D. M., \& Dodwell, E. R. (2014). Pediatric flatfoot: Cause, epidemiology, assessment, and treatment. Current Opinion in Pediatrics, 26(1), 93-100. https://doi.org/10.1097/MOP.0000000000000039

Duval, K., Lam, T., \& Sanderson, D. (2010). The mechanical relationship between the rear foot, pelvis and low-back. Gait \& Posture, 32(4), 637-640. https://doi.org/10.1016/j.gaitpost.2010.09.007

Evans, A. M., Copper, A. W., Scharfbillig, R. W., Scutter, S. D., \& Williams, M. T. (2003). Reliability of the Foot Posture Index and traditional measures of foot position. Journal of the American Podriatic Medical Association, 93(3), 203-213. https://doi.org/10.7547/87507315-93-3-203

Ezema, C. I., Abaraogu, U. O., \& Okafor, G. O. (2014). Flat foot and associated factors among primary school children: A cross-sectional study. Hong Kong Physiotherapy Journal, 32(1), 13-20. https://doi.org/10.1016/j.hkpj.2013.05.001

Farokhmanesh, K., Shirzadian, T., Mahboubi, M., \& Shahri, M. N. (2014). Effect of foot hyperpronation on lumbar lordosis and thoracic kyphosis in standing position using 3dimensional ultrasound-based motion analysis system. Global Journal of Health Science, 6(5), 254-260. https://doi.org/10.5539/gjhs.v6n5p254

Ghasemi, M. S., Koohpayehzadeh, J., Kadkhodaei, H., \& Ehsani, A. A. (2016). The effect of foot hyperpronation on spine alignment in standing position. Medical Journal of the Islamic Republic of Iran, 30: 466. PMID: 28491841

Giglio, C. A., \& Volpon, J. B. (2007). Development and evaluation of thoracic kyphosis and lumbar lordosis during growth. Journal of Children's Orthopaedics, 1(3), 187-193. https://doi.org/10.1007/s11832-007-0033-5

Khamis, S., \& Yizhar, Z. (2007). Effect of feet hyperpronation on pelvic alignment in a standing position. Gait \& Posture, 25(1), 127-134. https://doi.org/10.1016/j.gaitpost.2006.02.005

Khamis, S., Dar, G., Peretz, C., \& Yizhar, Z. (2015). The relationship between foot and pelvic alignment while standing. Journal of Human Kinetics, 46, 85-97. https://doi.org/10.1515/hukin-2015-0037

Legaye, J., Duval-Beaupère, G., Hecquet, J., \& Marty, C. (1998). Pelvic incidence: A fundamental pelvic parameter for three-dimensional regulation of spinal sagittal curves. European Spine Journal, 7(2), 99-103. https://doi.org/10.1007/s005860050038

Menz, H. (1998). Alternative techniques for the clinical assessment of foot posture. Journal of the American Podriatic Medical Association, 88(3), 119-129.

https://doi.org/10.7547/87507315-88-3-119 
Peeters, K., Schreuer, J., Burg, F., Behets, C., Van Bouwel, S., Dereymaeker, G., Sloten, J. V., \& Jonkers, I. (2013). Alterated talar and navicular bone morphology is associated with pes planus deformity: A CT-scan study. Journal of Orthopaedic Research, 31(2), 282-287. https://doi.org/10.1002/jor.22225

Pinto, R. Z., Souza, T. R., Trede, R. G., Kirkwood, R. N., Figueiredo, E. M., \& Fonseca, S. T. (2008). Bilateral and unilateral increases in calcaneal eversion affect pelvic alignment in standing position. Manual Therapy, 13(6), 513-519. https://doi.org/10.1016/j.math.2007.06.004

Predoiu, A. (2020). Metodologia cercetării ştiinţifice. Aspecte practice şi elemente de statistică neparametrică [Scientific research methodology. Practical aspects and elements of nonparametric statistics]. Discobolul.

Predoiu, A. (2021). Metodologia cercetării științifice. Statistică informatizată [Scientific research methodology. Computerised statistics]. Discobolul.

Redmond, A. C., Crosbie, J., \& Ouvrier, R. A. (2006). Development and validation of a novel rating system for scoring standing foot posture: The Foot Posture Index. Clinical Biomechanics, 21(1), 89-98. https://doi.org/10.1016/j.clinbiomech.2005.08.002

Tiberio, D. (2000). Relationship between foot pronation and rotation of the tibia and femur during walking. Foot \& Ankle International, 21(12), 1057-1060.

https://doi.org/10.1177/107110070002101214 